\title{
LACO-OFDM with Index Modulation for Optical Wireless Systems
}

\author{
Ali Waqar Azim, Yannis Le Guennec, Marwa Chafii, and Laurent Ros
}

\begin{abstract}
In this letter, we propose layered asymmetrically clipped optical-orthogonal frequency-division multiplexing (LACO-OFDM) with index modulation (IM), i.e., LACO-OFDMIM for optical wireless systems. In classical LACO-OFDM, the spectral leakage because of out-of-band clipping harmonics increases the baseband bandwidth (BB) resulting in lower spectral efficiencies. For LACO-OFDM-IM, we use IM and optimize the BB by filtering the out-of-band clipping harmonics. This amalgamation of IM and BB optimization leads to better spectral efficiency (SE). Simulation results shall affirm that LACO-OFDM-IM achieves higher SE, affords a comprehensive granularity for SE/energy efficiency trade-off, and outperforms classical alternatives in terms of bit-error-rate in both line-ofsight and time-dispersive channels.
\end{abstract}

Index Terms-Discrete Fourier transform, orthogonal frequency-division multiplexing, index modulation, intensitymodulation and direct-detection, optical wireless systems.

\section{INTRODUCTION}

I NTENSITY-modulation and direct-detection based opticalorthogonal frequency-division multiplexing (O-OFDM) techniques for optical wireless systems (OWS) have been comprehensively elucidated in the literature. Among these state-ofthe-art approaches, asymmetrically clipped O-OFDM (ACOOFDM) is widely recognized for its higher energy efficiency (EE) [1]. Albeit its high EE, the spectral efficiency (SE) is low because it only uses odd complex sub-carriers (CSCs) and suffers from baseband bandwidth (BB) enlargement resulting from out-of-band clipping harmonics. To augment the $\mathrm{SE}$ of ACO-OFDM, layered ACO-OFDM (LACO-OFDM) is proposed [2]. LACO-OFDM populates the idle CSCs of ACOOFDM by creating additional layers, where each succeeding layer populates the odd number CSCs left by the preceding layer. Thus, the number of CSCs reduces by a factor of two with each additional layer. This frame structure allows to increase the SE with each additional layer. Like ACOOFDM, the negative amplitude excursions in LACO-OFDM are eliminated by clipping the asymmetric bipolar time-domain (TD) signal at each layer. However, clipping results in out-ofband clipping harmonics, thus, LACO-OFDM's BB is twice relative to real bipolar pure frequency tone based signal. This aforesaid issue of bandwidth widening is circumvented by filtered ACO-OFDM (FACO-OFDM); which filters out-ofband clipping harmonics. FACO-OFDM can reduce the BB by up to $50 \%$ relative to ACO-OFDM. It is also highlighted that a variant of LACO-OFDM which optimizes the BB does not exist in the literature. Moreover, the classical approaches, such as ACO-OFDM, FACO-OFDM and LACO-OFDM do not offer granularity for SE/EE trade-off; which is important for

Ali Waqar Azim is with CEA Leti, MINATEC, 38054, Grenoble, France (email: aliwaqarazim@gmail.com)

Yannis Le Guennec and Laurent Ros are with Université Grenoble Alpes, CNRS, Institute of Engineering, Grenoble INP, GIPSA-LAB, 38000 Grenoble, France (email: \{yannis.le-guennec,laurent.ros\} @ grenoble-inp.fr).

Marwa Chafii is with ETIS, UMR 8051, CY Cergy Paris Université, ENSEA, CNRS, 95000 Cergy, France (email: marwa.chafii@ensea.fr) applications requiring varying $\mathrm{SE}$ and/or EE such as Internetof-Things (IoT), and optimization of system performance based on channel conditions.

From radio-frequency (RF) literature, it is gathered that index modulation (IM) enhances the SE and imparts granularity for SE/EE trade-off [3]. Inspired by these benefits, IM adaptations for O-OFDM are elucidated in [4], [5], [6]. [4] studies an amalgam of ACO-OFDM with IM (ACO-OFDMIM), whilst [6] investigates its enhanced variant, ACEOOFDM-IM. Nonetheless, these IM techniques also exhibit some shortcomings: (i) their BB is not optimal; and (ii) they are only energy-efficient for low alphabet cardinalities.

The proposed LACO-OFDM-IM uses the layering configuration, optimizes the $\mathrm{BB}$ and integrates IM to augment the SE and to impart granularity for SE/EE trade-off. For LACO-OFDM-IM, we adopt the IM precept of [6] which involves using real virtual sub-carriers (VSCs) as it leads to higher index-domain information. Moreover, we employ Pascal's Triangle (PT) based index mapping and de-mapping to reduce the overall system complexity (see [6] for details). A layered version of OFDM-IM (for RF) is studied in [7]. However, it is not compatible with OWS as the TD signal is neither non-negative nor real. Moreover, its frame structure is entirely different from the proposed LACO-OFDM-IM. Simulation results shall reveal the following concrete advantages of LACO-OFDM-IM over state-of-the-art counterparts: (i) higher achievable SE; (ii) comprehensive granularity for SE/EE tradeoff; and (iii) better EE; (iv) better bit-error-rate (BER) in lineof-sight (LOS) and time-dispersive channels.

We organize the rest of the article as follows. Section II presents the transceiver configuration. Section III compares the SE, SE/EE trade-off and the BER performance of LACOOFDM-IM with classical counterparts and the conclusions are rendered in Section IV.

\section{Transceiver Configuration of LACO-OFDM-IM}

\section{A. Transmitter Architecture}

Consider LACO-OFDM-IM transmitter with $\mathcal{L}$ layers and $N$ CSCs. For each symbol, the equiprobable bit sequence of length $\lambda$ is partitioned into $\mathcal{L}$ bit sequences each of length $\lambda_{l}$, where $l \in \llbracket 1, \mathcal{L} \rrbracket . \lambda_{l}$ is further split into $\lambda_{l}^{\mathrm{IM}} \mathrm{IM}$ bits, and $\lambda_{l}^{\mathrm{C}}$ constellation bits. At $l$ th layer, $N / 2^{l+1}$ CSCs or equivalently $N / 2^{l}$ real VSCs are available for IM [6]. $\lambda_{l}^{\mathrm{IM}}$ bits generate an integer, $Z_{l} \in \llbracket 0,2^{\lambda_{l}^{\mathrm{IM}}}-1 \rrbracket$, which identifies $\kappa_{l}$ VSCs to be activated among the $N / 2^{l}$ available. The indices of active sub-carriers form the sub-carrier activation pattern (SCAP) for the $l$ th layer, $\theta_{l, k}=\left\{\theta_{l, 1}, \theta_{l, 2}, \cdots, \theta_{l, \kappa}\right\}$, where $k \in \llbracket 1, \kappa_{l} \rrbracket$. By using PT based index mapping rather than combinatorial mapping, the complexity can be reduced $\mathcal{O}\left(2^{-l} N\right)$ from $\mathcal{O}\left(2^{-(l+1)} N^{2}\right)$. $\lambda_{l}^{\mathrm{C}}$ bits generate $\kappa_{l} M$-pulseamplitude modulation (PAM) alphabets, $\mathcal{X}_{l}[k]$ which ought to be modulated on the VSCs identified via $\theta_{l, k}$. Thus, the total number of bits transmitted per LACO-OFDM-IM symbol consisting of $\mathcal{L}$ layers of duration $T_{\mathrm{s}}$ is 


$$
\lambda^{\mathrm{LACO}-\mathrm{IM}}=\sum_{l=1}^{\mathcal{L}} \lambda_{l}=\sum_{l=1}^{\mathcal{L}} \lambda_{l}^{\mathrm{IM}}+\lambda_{l}^{\mathrm{C}},
$$

where the number of bits on the $l$ th layer is

$$
\lambda_{l}=\lambda_{l}^{\mathrm{IM}}+\lambda_{l}^{\mathrm{C}}=\left\lfloor\log _{2}\left(\begin{array}{c}
N / 2^{l} \\
\kappa_{l}
\end{array}\right)\right\rfloor+\log _{2}\left(M^{\kappa_{l}}\right),
$$

with $\lfloor\cdot\rfloor$ and $(\cdot)$ being the floor function and binomial coefficient, respectively.

Lemma 1. Considering $N C S C$ s and $M-P A M$, the maximum SE for LACO-OFDM-IM is attained when the number of activated VSCs per layer is approximately equal to

$$
\kappa_{l}^{\text {approx }} \approx\left\lfloor\frac{M N}{2^{l}(M+1)}\right\rfloor .
$$

Proof. Let us consider $\lambda_{l}$, for which, the following inequality must hold

$$
\lambda_{l} \leq \log _{2}\left(\begin{array}{c}
N / 2^{l} \\
\kappa_{l}
\end{array}\right)+\log _{2}\left(M^{\kappa_{l}}\right) .
$$

Taking the first derivative of (4) with respect to $\kappa_{l}$ leads to

$$
\frac{\mathrm{d} \lambda_{l}}{\mathrm{~d} \kappa_{l}} \leq \frac{\mathcal{H}_{N / 2^{l}-\kappa_{l}}-\mathcal{H}_{\kappa_{l}}+\log (M)}{\log (2)},
$$

where $\mathcal{H}_{m} \approx \log (m)+\varepsilon$ is the $m$ th harmonic number with $\varepsilon$ being the Euler-Mascheroni constant. Substituting $\mathrm{d} \lambda_{l} / \mathrm{d} \kappa_{l}=0$ and re-introducing the floor leads to (3).

Lemma 1 is attained owing to the log-concavity of the binomial coefficient. It may be noticed that there may be a marginal disparity between $\kappa_{l}$ and $\kappa_{l}^{\text {approx }}$ because $\kappa_{l}^{\text {approx }}$ is evaluated by ignoring the floor function. Moreover, closedform expressions developed in [8] can be used to determine the number of VSCs resulting in maximum SE. Nonetheless, these expressions also lead to approximate values of $\kappa_{l}$.

Using $\mathcal{X}_{l}[k]$ and $\theta_{l, k}$,we attain the VSCs vector, $S_{l}[\zeta]$ for $\zeta \in \llbracket 1, N / 2^{l} \rrbracket$ as

$$
S_{l}[\zeta]=\left\{\begin{array}{ll}
\sqrt{\left(1 / 2 \kappa_{l}\right)} \mathcal{X}_{l}[k], & k \in \theta_{l, k} \\
0, & \text { otherwise }
\end{array},\right.
$$

from which, we obtain $\dot{X}_{l}[\gamma]=S_{l}[\gamma]+j S_{l}\left[\gamma+N / 2^{l+1}\right]$, where $\gamma \in \llbracket 1, N / 2^{l+1} \rrbracket$ and $j^{2} \triangleq-1$. For $l$ th layer, we have $N / 2^{l+1}$ elements $\dot{X}_{l}[\gamma]$ from the signal space [6]; which are modulated onto the $N / 2^{l+1}$ CSCs of the same layer. The $l$ th layer frequency-domain (FD) symbol, over-sampled by $L$ comprising of $N$ CSCs is $X_{l}[\sigma]$ for $\sigma \in \Omega=\llbracket-L N / 2, L N / 2-1 \rrbracket$. An over-sampling factor of $L \geq 4$ is needed for the envelop characteristics of O-OFDM (IM/non-IM) to converge [9]. The FD symbol conforming to Hermitian symmetry is given as

$$
X_{l}[\sigma]= \begin{cases}\dot{X}_{l}[\gamma] & \sigma=\sigma^{+} \in \Omega^{+} \\ \dot{X}_{l}^{*}[\gamma] & \sigma=\sigma^{-} \in \Omega^{-} \\ 0 & \text { otherwise }\end{cases}
$$

Let $\mu(\gamma, l)=2^{l-1}[2(\gamma-1)+1]$, then $\sigma^{+}=\mu(\gamma, l) \in$ $\Omega^{+} \in \llbracket 0, N / 2 \rrbracket$ and $\sigma^{-}=-\mu(\gamma, l) \in \Omega^{-}=\llbracket-1,-N / 2 \rrbracket$. Moreover, $(\cdot)^{*}$ is the complex conjugate. Subsequently, using $L N$-order inverse fast Fourier transform (IFFT), $X_{l}[\sigma]$ is transformed to a real bipolar TD signal, $x_{l}^{\mathrm{BP}}(n)$ for $n \in$ $\llbracket 1, L N \rrbracket$, which adheres to the anti-symmetric property, i.e.,
$x_{l}^{\mathrm{BP}}(p)=-x_{l}^{\mathrm{BP}}(p+L N / 2)$ with $p \in \llbracket 1, L N / 2 \rrbracket$. Subsequently, $x_{l}^{\mathrm{BP}}(n)$ is clipped to attain real non-negative TD signal, $x_{l}^{\text {clip }}(n)=x_{l}^{\mathrm{D}}(n)+x_{l}^{\mathrm{C}}(n)$, where $x_{l}^{\mathrm{D}}(n)=0.5 x_{l}^{\mathrm{BP}}(n)$ is the TD data carrying signal, and $x_{l}^{\mathrm{C}}(n)=0.5\left|x_{l}^{\mathrm{BP}}(n)\right|$ is the TD clipping distortion. The out-of-band clipping, i.e., $\left.X_{l}^{\mathrm{C}} \sigma\right]=\operatorname{IFFT}\left\{x_{l}^{\mathrm{C}}(n)\right\}$ cause spectral leakage, thus, the BB for $x_{l}^{\text {clip }}(n)$ is approximately $B^{\text {ext }} \approx N / T_{\mathrm{s}}$, which is twice the $\mathrm{BB}$ required by a real bipolar pure frequency tones based signal, i.e., $B^{\text {opt }} \approx N / 2 T_{\mathrm{s}}$. Once the $\mathcal{L}$ layers are populated, the clipped composite TD signal for $\mathcal{L}$ layers is attained as

$$
x^{\operatorname{comp}}(n)=\sum_{l=1}^{\mathcal{L}} x_{l}^{\text {clip }}(n) .
$$

To optimize the BB, $x^{\operatorname{comp}}(n)$ is transformed to the FD counterpart, $X^{\text {comp }}[\sigma]$ using $L N$-order FFT. $X^{\text {comp }}[\sigma]$ is then passed through a low-pass filter having bandwidth $B^{\mathrm{F}}=$ $(N+2 \alpha-1) / T_{\mathrm{s}}$, resulting in $X^{\mathrm{F}}[\sigma]=X^{\mathrm{comp}}[\sigma] \times H^{\mathrm{F}}[\sigma]$, where $\alpha$ is so-called tunability factor of the low-pass filter, and $H^{\mathrm{F}}[\sigma]$ is a rectangular window low-pass filter, i.e., $H^{\mathrm{F}}[\sigma]=1$ for $\sigma \in \llbracket-N / 2-\alpha, N / 2+\alpha-1 \rrbracket$ and zero otherwise, where $\alpha \in \llbracket 0,(L-1)^{N} / 2 \rrbracket$. For $B^{\mathrm{F}}$, the resulting $\mathrm{BB}$ required for $X^{\mathrm{F}}[\sigma]$ is $B \approx(N / 2+\alpha) / T_{\mathrm{s}} . X^{\mathrm{F}}[\sigma]$ when converted to $x^{\mathrm{F}}(n)$ using $L N$-order IFFT exhibits peak regrowth in negative amplitude excursions, thus, a bias $\beta=\left|\min x^{\mathrm{F}}(n)\right|$ is added to $x^{\mathrm{F}}(n)$ resulting in $x^{+}(n)=x^{\mathrm{F}}(n)+\beta$. The average electrical symbol energy of $x^{+}(n)$ is scaled to unity, i.e., $E_{\mathrm{s}_{(\text {elec }}}=\mathbb{E}\left\{\left|x^{+}(n)\right|^{2}\right\}=\sum_{n}\left|x^{+}(n)\right|^{2}=1$, where $\mathbb{E}\{\cdot\}$ evaluates the ensemble average. After digital-to-analog conversion of $x^{+}(n)$, an intensity waveform, $x(t) \geq 0$ is attained and is transmitted to the optical wireless channel.

Relative to classical LACO-OFDM, the proposed LACOOFDM-IM requires an additional $L N$-order IFFT and $L N$ order FFT to filter out-of-band clipping harmonics.

\section{B. Receiver Architecture}

The received photo-detected waveform is $y(t)$, which after analog-to-digital conversion is processed by $L N$-order FFT to yield $Y[\sigma]$. After zero-forcing equalization, we attain

$$
\hat{Y}[\sigma]=\sum_{l=1}^{\mathcal{L}} \hat{X}_{l}^{\mathrm{D}}[\sigma]+\hat{X}_{l}^{\mathrm{C}}[\sigma]
$$

where $\hat{X}_{l}^{\mathrm{D}}[\sigma]$ and $\hat{X}_{l}^{\mathrm{C}}[\sigma]$ are the FD counterparts of $x_{l}^{\mathrm{D}}(n)$ and $x_{l}^{\mathrm{C}}(n)$, respectively. (9) implies that to correctly detect the transmit information, the clipping distortion has to be estimated and removed, such that for the lth layer, we have

$$
\hat{Y}_{l}[\sigma]=\hat{Y}_{(l-1)}[\sigma]-\hat{X}_{l}^{\mathrm{C}}[\sigma] .
$$

For $l=1$, the clipping distortion do not fall on the data bearing CSCs, thus, $\hat{X}_{1}^{\mathrm{C}}[\sigma]=0$ and $\hat{Y}_{1}[\sigma]=\hat{Y}_{0}[\sigma]=\hat{Y}[\sigma]$. For $l>1$, the clipping distortion of $(l-1)$ th layer falling on $l$ th layer, i.e., $\hat{X}_{l}^{\mathrm{C}}[\sigma]$ has to be eliminated. Note that $\hat{X}_{l}^{\mathrm{C}}[\sigma]$ can be forthrightly estimated once the SCAP, $\hat{\theta}_{(l-1), k}$ and $\hat{\mathcal{X}}_{(l-1)}[k]$ are determined. In the sequel, we elaborate on how $\hat{\mathcal{X}}_{l}[k]$ and $\hat{\theta}_{l, k}$ for the $l$ th layer can be attained.

The data bearing CSCs for the $l$ th layer $\dot{Y}_{l}[\gamma]=2 \hat{Y}_{l}[\mu(l, \gamma)]$ are used to determine the alphabets on the VSCs as $\ddot{Y}_{l}[\zeta]=$ $\left[\Re\left\{\dot{Y}_{l}[\gamma]\right\}, \Im\left\{\dot{Y}_{l}[\gamma]\right\}\right]$, where $\Re\{\cdot\}$ and $\Im\{\cdot\}$ extract the real 
TABLE I: Spectral efficiencies in bits/s/Hz of the studied approaches.

\begin{tabular}{c|c}
\hline \hline Approach & Spectral Efficiency (bits/s/Hz) \\
\hline \hline LACO-OFDM-IM & {$\left[\sum_{l=1}^{\mathcal{L}}\left[\log _{2}\left(\begin{array}{c}N / 2^{l} \\
\kappa_{l}\end{array}\right)\right\rfloor+\log _{2}\left(M^{\kappa_{l}}\right)\right](N / 2+\alpha)^{-1}$} \\
ACEO-OFDM-IM & {$\left[\left\lfloor\log _{2}\left(\begin{array}{c}N / 2 \\
\kappa\end{array}\right)\right\rfloor+\log _{2}\left(M^{\dot{\kappa}}\right)\right] N^{-1}$} \\
ACO-OFDM-IM & {$\left[\left\lfloor\log _{2}\left(\begin{array}{c}N / 4 \\
\tilde{\kappa}\end{array}\right)\right\rfloor+\log _{2}\left(\tilde{M}^{\tilde{\kappa}}\right)\right] N^{-1}$} \\
LACO-OFDM & {$\left[\sum_{l=1}^{\mathcal{L}} N / 2^{l+1} \log _{2}(\tilde{M})\right] N^{-1}$} \\
FACO-OFDM & $\left.\left[(N / 4) \log _{2}(\tilde{M})\right](N / 2+\alpha)\right)^{-1}$ \\
ACO-OFDM & {$\left[(N / 4) \log _{2}(\tilde{M})\right] N^{-1}$} \\
\hline \hline
\end{tabular}

and imaginary components of a complex number, respectively. Hereby, we use sub-optimal energy detector (ED) to determine the SCAP, $\hat{\theta}_{l, k}$. To do so, we evaluate the energies of the VSCs as $\xi_{l, \zeta}=\left|\hat{\bar{Y}}_{l}[\zeta]\right|^{2}$. Note that the activated VSCs have higher energies relative to the inactivate ones, thus, $\kappa_{l}$ highest energies are retained, i.e., $\xi_{l, k}$. The indices of these $\kappa_{l}$ VSCs sorted in descending order form the SCAP, $\hat{\theta}_{l, k}$, from which, $\lambda_{l}^{\mathrm{IM}}$ is determined. Subsequently, the $M$-PAM alphabets modulated onto the activated VSCs are attained as $\hat{\mathcal{X}}_{l}[k]=\hat{\ddot{Y}}_{l}\left[\hat{\theta}_{l, k}\right]$, using which, $\hat{\lambda}_{l}^{\mathrm{C}}$ are attained. $\hat{\mathcal{X}}_{l}[k]$ and $\hat{\theta}_{l, k}$ are then used to estimate the clipping distortion on the succeeding layer, i.e., $\hat{X}_{(l+1)}^{\mathrm{C}}[\sigma]$. This process continues until all the $\mathcal{L}$ layers are processed.

The receiver complexity of LACO-OFDM-IM in terms of required IFFT/FFT operations is same as that of classical LACO-OFDM.

\section{Performance Analysis of LACO-OFDM-IM}

\section{A. Spectral Efficiency Analysis}

LACO-OFDM-IM and ACEO-OFDM-IM use $M$-PAM, whereas, ACO-OFDM-IM, LACO-OFDM, FACO-OFDM and ACO-OFDM employ $\tilde{M}$-quadrature-amplitude modulation (QAM). The number of active VSCs for LACO-OFDM-IM and ACEO-OFDM-IM are $\sum_{l} \kappa_{l}$ and $\dot{\kappa}$, respectively, whereas, the number of activated CSCs in ACO-OFDM-IM is $\tilde{\kappa}$. We consider $N$ CSCs, a symbol duration of $T_{\mathrm{s}}$, and $\mathcal{L}$ layers for the layered approaches. It is recalled that the BB of LACOOFDM-IM and FACO-OFDM is $B$, whereas, for the remaining approaches it is $B^{\text {ext }}$. In the sequel, we consider $\alpha=0$ for LACO-OFDM-IM and FACO-OFDM. Taking into account the BB and the frame structure of each approach, we calculate the spectral efficiencies, which are summarized in Table. I.

Considering $N=32$ and $\mathcal{L}=3$, the evolution of spectral efficiencies of the IM approaches with respect to the $\sum_{l} \kappa_{l}, \dot{\kappa}$ and $\tilde{\kappa}$ is presented in Fig. 1, from which, we observe that LACO-OFDM-IM is capable of attaining high spectral efficiencies using $M=2$. Same $\mathrm{SE}$ trend is expected for higher $M$. On the contrary, all the alternatives require significantly higher modulation alphabets to attain the same SE. The SE of LACO-OFDM-IM is between the range of $[0.3125,2.375] \mathrm{bits} / \mathrm{s} / \mathrm{Hz}$ for $\sum_{l} \kappa_{l}=17$ and $M=2$. It is essential to highlight here that even though the $l$ th layer has $N / 2^{l}$ VSCs available for IM, the number of active VSCs are $\kappa_{l}<N / 2^{l}$. For maximum SE, we attain that $\left\{\kappa_{1}^{\text {approx }}, \kappa_{2}^{\text {approx }}, \kappa_{3}^{\text {approx }}\right\}=\{10,5,2\}$ using (3), whereas

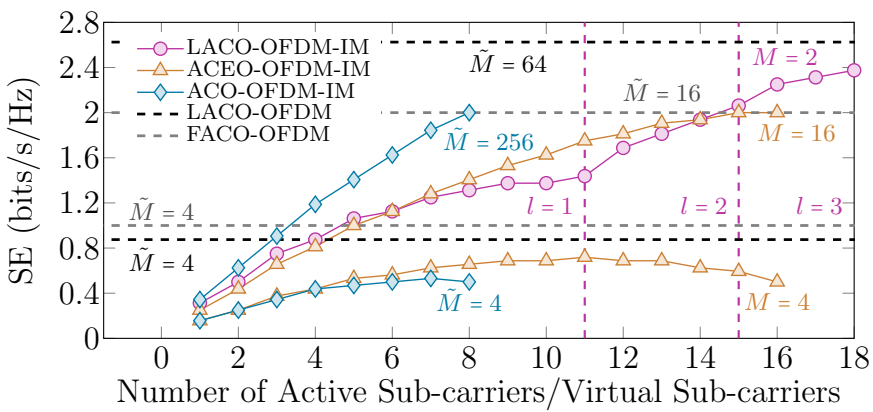

Fig. 1: (Color version online) The evolution of SE versus the $\kappa, \dot{\kappa}$ and $\tilde{\kappa}$ for LACO-OFDM-IM, ACEO-OFDM-IM and ACO-OFDM-IM, respectively for $N=32$. The spectral efficiencies of LACO-OFDM and FACO-OFDM (with $\alpha=0$ ) are also provided as benchmarks.

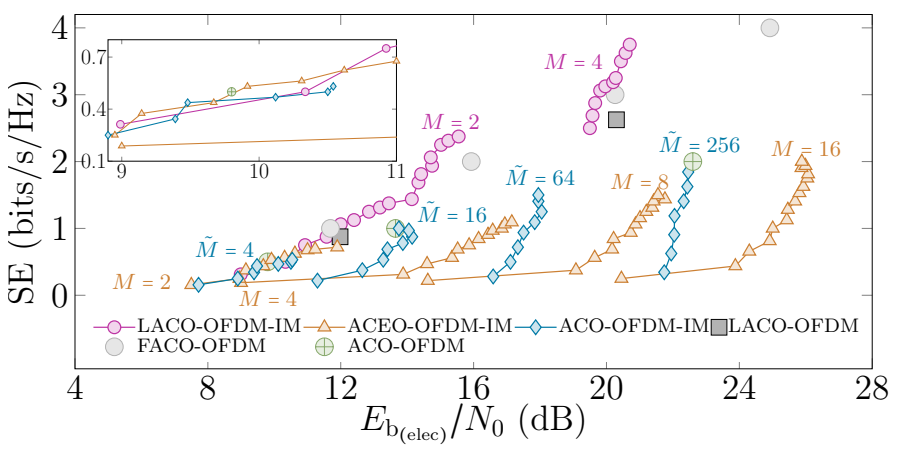

Fig. 2: (Color version online) SE/EE trade-off for LACO-OFDM-IM using $\{N, L, \alpha\}=\{32,4,0\}$ for BER of $10^{-3}$.

the optimal values are $\left\{\kappa_{1}, \kappa_{2}, \kappa_{3}\right\}=\{11,4,2\}$. This implies that when $\kappa_{l}>11$ for $l=1$, the VSCs of $l=2$ should be modulated and so on so forth. We observe a considerable improvement over the conventional LACO-OFDM, for which, the SE of $0.875 \mathrm{bits} / \mathrm{s} / \mathrm{Hz}$ and $2.625 \mathrm{bits} / \mathrm{s} / \mathrm{Hz}$ is attained using $\tilde{M}=4$ and $\tilde{M}=64$, respectively. This implies that LACOOFDM requires $\tilde{M}=64$ to attain a SE close to the maximum achievable SE of LACO-OFDM-IM. FACO-OFDM requires alphabets of lesser cardinalities relative to other approaches because of optimized BB. Moreover, for a given $\tilde{M}$, the attainable SE of ACO-OFDM (not plotted in Fig. 1) is half of $\mathrm{SE}$ of FACO-OFDM. Lastly, the granularity for SE/EE tradeoff for IM approaches is also observable from Fig. 1.

\section{B. Spectral-Energy Efficiency Analysis}

Fig. 2 depicts the SE/EE trade-off and granularity of LACOOFDM-IM in LOS channel. Note that for LACO-OFDMIM, the spectral efficiencies between $[0.3125,2.375]$ bits $/ \mathrm{s} / \mathrm{Hz}$ are attained using $\{M, \alpha\}=\{2,0\}$ and $\left\{\kappa_{1}, \kappa_{2}, \kappa_{3}\right\}=$ $\{11,4,2\}$, whilst the spectral efficiencies between the range $[2.5,3.75]$ bits $/ \mathrm{s} / \mathrm{Hz}$ are achieved when $\{M, \alpha\}=\{4,0\}$ and $\left\{\kappa_{1}, \kappa_{2}, \kappa_{3}\right\}=\{11,7,3\}$. The EE is determined by evaluating the electrical signal-to-noise ratio per bit $E_{\mathrm{b}_{(\text {elec }}} / N_{0}$ required to attain a BER of $10^{-3} \cdot E_{\mathrm{b}_{(\text {elec }}} / N_{0}$ is equal to $E_{\mathrm{s}_{(\text {elec }}} T_{\mathrm{s}} /\left(N_{0} \lambda^{\mathrm{LACO}-\mathrm{IM}}\right)$, where $N_{0}$ is the mono-lateral noise spectral density. For a fair comparison, we use the sub-optimal ED receiver for all the IM approaches. The IM approaches generally lose their EE when alphabet cardinality increases. Interestingly, for LACO-OFDM-IM, an increase in alphabet cardinality affects the EE but it still remains the most 
energy-efficient approach compared to other counterparts. For example, we observe $4 \mathrm{~dB}$ penalty for FACO-OFDM at SE of $4 \mathrm{bits} / \mathrm{s} / \mathrm{Hz}(\tilde{M}=256)$ relative to LACO-OFDM-IM when $\mathrm{SE}$ is $3.75 \mathrm{bits} / \mathrm{s} / \mathrm{Hz}\left(\left\{M, \sum_{l} \kappa_{l}\right\}=\{4,21\}\right)$. Thus, from Fig. 2 , we observe the following advantages of LACO-OFDM-IM over other alternatives: (i) it is the most energy-efficient and spectral-efficient approach not only for low spectral efficiencies but also for higher spectral efficiencies; and (ii) it provides the most comprehensive granularity for SE/EE trade-off for wider SE range which is impossible with classical ACOOFDM, FACO-OFDM and LACO-OFDM. More importantly, LACO-OFDM-IM is significantly energy-efficient than its direct state-of-the-art counterpart LACO-OFDM particularly for spectral efficiencies $>1 \mathrm{bits} / \mathrm{s} / \mathrm{Hz}$. It is reiterated that the classical IM approaches require alphabets of higher cardinalities to attain the same peak SE as that of LACO-OFDM-IM and thus are not energy-efficient.

\section{Bit-Error Rate Performance Analysis}

The BER performance of LACO-OFDM-IM in LOS channel and time dispersive channel for the reference SE of $2.375 \mathrm{bits} / \mathrm{s} / \mathrm{Hz}$ is presented in Fig. 3. In fact, this reference is the maximum achievable SE of LACO-OFDM-IM for $\left\{\sum_{l} \kappa_{l}, M, \alpha\right\}=\{17,2,0\}$. For ACEO-OFDM-IM and ACO-OFDM-IM, the SE close to the reference SE is 2 bits $/ \mathrm{s} / \mathrm{Hz}$, which is achieved for the respective approaches for $\{M, \dot{\kappa}\}=\{16,15\}$ and $\{\tilde{M}, \tilde{\kappa}\}=\{256,8\}$. Moreover, we use $\mathcal{L}=2$ and $\tilde{M}=64$ which results in SE of $2.25 \mathrm{bits} / \mathrm{s} / \mathrm{Hz}$ for LACO-OFDM. Lastly, $\tilde{M}=256$ and $\{\tilde{M}, \alpha\}=\{16,0\}$ is used for ACO-OFDM and FACOOFDM, respectively which also result in SE of 2 bits $/ \mathrm{s} / \mathrm{Hz}$. Considering data rate of $200 \mathrm{Mbits} / \mathrm{s}$, a delay spread of $10 \mathrm{~ns}$, the time-dispersive optical wireless channel is emulated using ceiling bounce model [10]. Moreover, we consider both lowcomplexity sub-optimal ED and maximum likelihood detector (MLD) for LACO-OFDM-IM. The MLD complexity for $l$ th layer is $\mathcal{O}\left(2^{l} N^{-1}\left[2^{\lambda_{l}^{\mathrm{IM}}} M^{\kappa_{l}}+\kappa_{l} M\right]\right)$ [6], whereas, the ED complexity is $\mathcal{O}\left(2^{-l} N\right)$. From Fig. 3, we observe that the BER performance of LACO-OFDM-IM is considerably better than classical counterparts (IM/non-IM) in both LOS and time dispersive channels with MLD exhibiting marginally better performance than ED. Note that the BER performance of ACO-OFDM-IM and ACO-OFDM is the same because all the CSCs in ACO-OFDM-IM are activated to attain 2 bits $/ \mathrm{s} / \mathrm{Hz}$, thus, its frame structure is exactly the same as ACO-OFDM. It can also be observed that the BER performance of FACOOFDM is closest to that of LACO-OFDM-IM, however, in the portrayed scenario, the SE of LACO-OFDM-IM is approximately $16 \%$ higher than the SE of FACO-OFDM. Note that for lower $E_{\mathrm{b}_{(\text {elec }}} / N_{0}$, the probability of correct detection of the active indices of VSCs is less, which, by extension also results in incorrect detection of $M$-PAM alphabets leading to higher BER. As $E_{\mathrm{b}_{(\text {elec })}} / N_{0}$ increases, the probability of correct detection of active indices and $M$-PAM alphabets improves resulting in reduction of BER. One of the reason for better BER performance of LACO-OFDM-IM is the requirement of lower alphabet cardinalities, whilst other alternatives require alphabets of significantly higher cardinality.
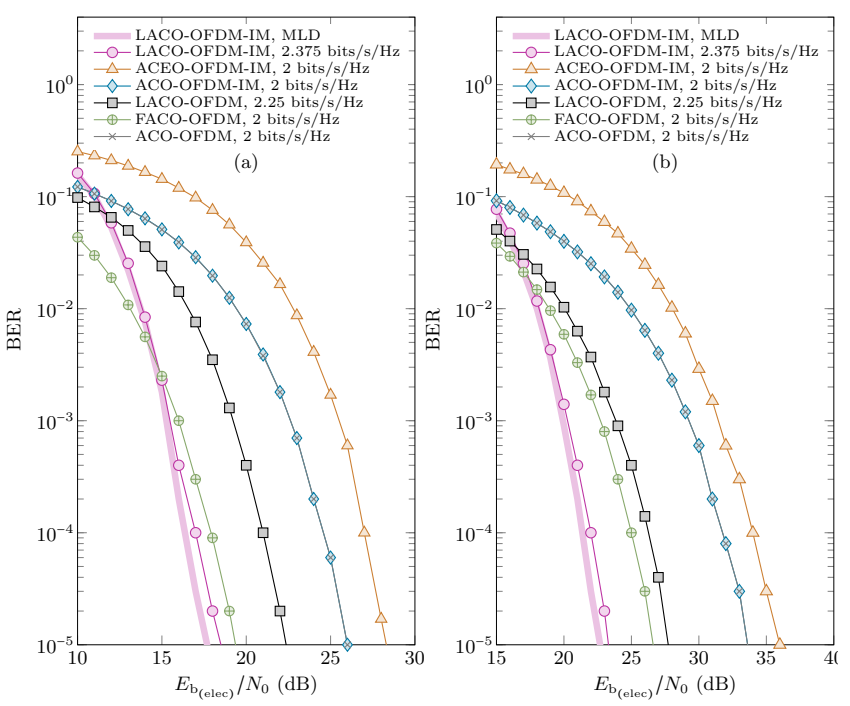

Fig. 3: (Color version online) BER performance comparison of LACOOFDM-IM with classical counterparts for reference SE of $2.375 \mathrm{bits} / \mathrm{s} / \mathrm{Hz}$ in (a) LOS and (b) time-dispersive channel using $N=32$.

\section{CONCLUSIONS}

In this letter, we propose LACO-OFDM-IM which employs the layering architecture, IM and BB optimization to provide significant SE gains relative to classical benchmarks. We observe that in addition to being the most energy-efficient than counterparts, LACO-OFDM-IM is capable of achieving higher spectral efficiencies and provides comprehensive granularity for SE/EE trade-off. We also analytically determine the approximate number of VSCs which maximize the SE. Moreover, the BER performance of LACO-OFDM-IM is better than classical counterparts in both LOS and time dispersive channels. These advantages of LACO-OFDM-IM portrayed herein, makes it a viable alternative to other counterparts.

\section{REFERENCES}

[1] J. Armstrong and A. J. Lower. Power efficient optical OFDM. Electron. Lett, 42(6):370-372, 2006.

[2] Q. Wang, C. Qian, X. Guo, Z. Wang, D. G. Cunningham, and I. H. White. Layered ACO-OFDM for intensity-modulated direct-detection optical wireless transmission. Optics Express, 23(9):12382-12393, 2015.

[3] M. Wen, B. Zheng, K. J. Kim, M. Di Renzo, T. A. Tsiftsis, K. Chen, and N. Al-Dhahir. A survey on spatial modulation in emerging wireless systems: Research progresses and applications. IEEE J. Sel. Areas Commun., 37(9):1949-1972, 2019.

[4] E. Başar and E. Panayırcı. Optical OFDM with index modulation for visible light communications. In Intl. Wksp. Opt. Wireless Commun., pages 11-15, 2015.

[5] A. W. Azim, M. Chafii, Y. Le Guennec, and L. Ros. Spectral and energy efficient fast-OFDM with index modulation for optical wireless systems. IEEE Commun. Lett., 24(8):1771-1774, 2020.

[6] A. W. Azim, Y. Le Guennec, M. Chafii, and L. Ros. Enhanced opticalOFDM with index and dual-mode modulation for optical wireless systems. IEEE Access, 8:128646-128664, 2020.

[7] J. Li, S. Dang, M. Wen, X.-Q. Jiang, Y. Peng, and H. Hai. Layered orthogonal frequency division multiplexing with index modulation. IEEE Systems Journal, 13(4):3793-3802, 2019.

[8] W. Li, H. Zhao, C. Zhang, L. Zhao, and R. Wang. Generalized selecting sub-carrier modulation scheme in ofdm system. In IEEE ICC, pages 907-911, 2014.

[9] S. Mazahir, A. Chaaban, H. Elgala, and M.-S. Alouini. Achievable rates of multi-carrier modulation schemes for bandlimited IM/DD systems. IEEE Trans. Wireless Commun., 18(3):1957-1973, 2019.

[10] J. B. Carruthers and J. M. Kahn. Modeling of nondirected wireless infrared channels. IEEE Trans. Commun., 45(10):1260-1268, 1997. 